\title{
Not All Particles Are Equal: The Selective Enrichment of Particle-Associated Bacteria from the Mediterranean Sea
}

\section{OPEN ACCESS}

Edited by:

Marcelino T. Suzuki,

Sorbonne Universities and CNRS,

France

Reviewed by:

Byron C. Crump,

Oregon State University, USA

Uli Stingl,

King Abdullah University of Science

and Technology, Saudi Arabia

${ }^{*}$ Correspondence:

Francisco Rodriguez-Valera frvalera@umh.es

${ }^{\dagger}$ Present Address: Nikole E. Kimes,

Division of Gastroenterology, Department of Medicine, University of California,

San Francisco, San Francisco, CA,

USA

${ }^{\ddagger}$ These authors have contributed equally to this work.

Specialty section:

This article was submitted to

Aquatic Microbiology,

a section of the journal

Frontiers in Microbiology

Received: 10 February 2016 Accepted: 09 June 2016

Published: 22 June 2016

Citation:

López-Pérez M, Kimes NE

Haro-Moreno JM and

Rodriguez-Valera F (2016) Not All Particles Are Equal: The Selective

Enrichment of Particle-Associated Bacteria from the Mediterranean Sea.

Front. Microbiol. 7:996.

doi: 10.3389/fmicb.2016.00996

\section{Mario López-Pérez ${ }^{\ddagger}$, Nikole E. Kimes ${ }^{\dagger \neq}$, Jose M. Haro-Moreno and Francisco Rodriguez-Valera *}

Evolutionary Genomics Group, División de Microbiología, Universidad Miguel Hernández, Alicante, Spain

We have used two metagenomic approaches, direct sequencing of natural samples and sequencing after enrichment, to characterize communities of prokaryotes associated to particles. In the first approximation, different size filters $(0.22$ and $5 \mu \mathrm{m})$ were used to identify prokaryotic microbes of free-living and particle-attached bacterial communities in the Mediterranean water column. A subtractive metagenomic approach was used to characterize the dominant microbial groups in the large size fraction that were not present in the free-living one. They belonged mainly to Actinobacteria, Planctomycetes, Flavobacteria and Proteobacteria. In addition, marine microbial communities enriched by incubation with different kinds of particulate material have been studied by metagenomic assembly. Different particle kinds (diatomaceous earth, sand, chitin and cellulose) were colonized by very different communities of bacteria belonging to Roseobacter, Vibrio, Bacteriovorax, and Lacinutrix that were distant relatives of genomes already described from marine habitats. Besides, using assembly from deep metagenomic sequencing from the particle-specific enrichments we were able to determine a total of 20 groups of contigs (eight of them with $>50 \%$ completeness) and reconstruct de novo five new genomes of novel species within marine clades ( $>79 \%$ completeness and $<1.8 \%$ contamination). We also describe for the first time the genome of a marine Rhizobiales phage that seems to infect a broad range of Alphaproteobacteria and live in habitats as diverse as soil, marine sediment and water column. The metagenomic recruitment of the communities found by direct sequencing of the large size filter and by enrichment had nearly no overlap. These results indicate that these reconstructed genomes are part of the rare biosphere which exists at nominal levels under natural conditions.

Keywords: metagenomics, microbial diversity, particle-attached bacteria, free-living bacteria, phages, Mediterranean Sea, particle-enrichments

\section{INTRODUCTION}

Characterizing the true diversity of microbes in nature is a daunting task, yet great advances have been achieved through the use of high throughput sequencing (Sogin et al., 2006; Allen et al., 2012; Smith et al., 2013; Dupont et al., 2015). Next generation sequencing (NGS), in particular, has been extremely informative because it permits a glimpse into microbial diversity 
with very little bias and with an in depth view of the microbes that only the complete genome can provide (Temperton and Giovannoni, 2012). As a result, studying natural communities by direct sequencing has become common and has provided new insights into the diversity of microbes in many environments (Qin et al., 2010; Mackelprang et al., 2011; Fierer et al., 2012; DeLong, 2013). The potential for combining sequencing based studies (metagenomics) with that of culture based studies (enrichments), however, remains relatively unexploited. This combined approach provides a powerful tool to answer many biological queries, including the role of particulate matter in shaping marine microbial diversity, as we have done here.

An exciting transformation has occurred in our understanding of the oceans' influence on nutrient cycling over the past two decades, and long gone are the days when marine microorganisms were largely discounted as contributors to this process (Azam, 1998). Instead, we now recognize the oceans' microbial communities as important regulators of major oceanic biogeochemical cycles, capable of broad impacts (Jiao and Azam, 2011). Originally thought to simply assimilate and respire dissolved organic material (DOM), current evidence shows that many marine microorganisms actively sense, seek and transform particulate organic matter (POM) through various processes (Azam and Malfatti, 2007). This is an important contribution to ocean ecosystems that are largely oligotrophic environments containing patches of POM in the form of clustered and sinking particles, including detritus and marine snow (Azam and Long, 2001; Kiørboe and Jackson, 2001; Azam and Malfatti, 2007). Furthermore, genomic studies over the past decade have provided enlightening insights into the many mechanisms utilized by specific marine microbes in ocean ecosystem functioning (Azam and Worden, 2004; Azam and Malfatti, 2007; Allen et al., 2013).

Not all marine microorganisms are necessarily capable of sensing POM and subsequently moving to the source and transforming it into dissolved nutrients. Rather it has been proposed that there are two distinct communities in marine ecosystems: the particle-associated fraction, which is capable of colonizing POM and producing DOM, and the free-living fraction that thrives on the DOM released by the particle colonizers (Azam and Long, 2001; Kiørboe and Jackson, 2001; Allen et al., 2013). The greatest emphasis of marine microbial research has previously been on the free-living microorganisms (DeLong et al., 2006; Rusch et al., 2007; Dinsdale et al., 2008; Ghai et al., 2010), in large part due to the fact that many of the most abundant marine bacteria, e.g., Pelagibacter ubique (Giovannoni et al., 2005) and Prochlorococcus spp. (Rocap et al., 2003), are thought to be free-living. There is a large amount of evidence, however, providing support for the existence of particle-associated (PA) communities distinct from their freeliving (FL) cohorts (Allen et al., 2013; Rieck et al., 2015). To date, only three direct sequencing metagenomic studies have been published, in which the differences in microbial community composition between presumably FL and PA communities based on size fractions. All three have focused on microbial communities in the Pacific Ocean, studying the coastal and open ocean waters off the Californian coast (Allen et al., 2012), the Columbia River coastal margin in the Pacific Northwest (Smith et al., 2013), and the oxygen minimum zone off the Chilean coast (Ganesh et al., 2014). These studies revealed a distinct taxonomic classification represented by an increased association with eukaryotes and viruses in the larger fractions, as well as, differences in functional classification with an increase in gene diversity associated with particle-associated fractions. These studies and also earlier studies based on $16 \mathrm{~S}$ rRNA gene sequencing (DeLong et al., 1993; Crump et al., 1999) showed that the compositions of the PA communities were related to members of the Bacteroidetes (considered to be specialists in the degradation of high molecular weight organic matter), Plactomycetes and Proteobacteria (Gamma and Deltaproteobacteria). Recently, a combination of metagenome and metatrancriptome methodologies was used to analyze carbon and nutrient flux through free-living and particleassociated microbes of the Amazon Plume (Satinsky et al., 2014).

In the study presented here, we combine direct metagenomic sequencing with culture-based enrichments to explore the role of particles in shaping microbial diversity in the Mediterranean Sea. To initially characterize the size-fractionated microbial communities of the Mediterranean, we sequenced and analyzed two large metagenomic datasets of small $(0.22-5.0 \mu \mathrm{m})$ and large (5.0-20.0 $\mathrm{n} \mu \mathrm{m})$ filter fractions theoretically representing the FL and PA communities, respectively. We also investigated the selective impact of different particles on community composition using metagenomic sequencing of particle-specific enrichments. Our results confirm that the Mediterranean FL and PA microbial communities are distinct. Moreover, we show that different particle chemical composition and structure can drive the enrichment of distinct and specific particle-associated community structures. In addition, using assembly from deep metagenomic sequencing from the particle-specific enrichments, we describe several nearly complete genomes most of them belonging to new species. These reconstructed genomes are part of the rare biosphere, which exists at nominal levels under natural conditions.

\section{MATERIALS AND METHODS}

\section{Sample Collection}

For the free-living (FL, 0.22-5.0 $\mu \mathrm{m}$ fraction) and particleassociated (PA, 5.0-20.0 $\mu \mathrm{m}$ fraction) metagenomes, $200 \mathrm{~L}$ of Mediterranean seawater was collected off the coast of Alicante, Spain $\left(38^{\circ} 4^{\prime} 6.64^{\prime \prime} \mathrm{N} / 0^{\circ} 13^{\prime} 55.18^{\prime \prime} \mathrm{W}\right)$ on September 6th 2013 and January 27 th 2015 from 55 and $20 \mathrm{~m}$ depth, respectively $\left(16^{\circ} \mathrm{C}\right)$. The seawater was sequentially filtered using 20, 5, and 0.22 $\mu \mathrm{m}$ filters (GCWP2932A, Durapore, Millipore, Billerica, MA, USA), and the filters were immediately frozen at $-20^{\circ} \mathrm{C}$ until processed for DNA extraction. For the particle-enriched (PE) metagenomes, seawater was also collected off the coast of Alicante, Spain $\left(38^{\circ} 16^{\prime} 42^{\prime \prime} \mathrm{N} / 00^{\circ} 14^{\prime} 39^{\prime \prime} \mathrm{W}\right)$ on May 20 th 2013.

\section{Particle-Enrichments}

In the laboratory, $480 \mathrm{~mL}$ of seawater from $55 \mathrm{~m}$ depth was added to each of five flasks (2L) containing $50 \mathrm{~g}$ of one of 
the following: 1. seawater from the above sampling-control (SW), 2. Sand (SWsnd, Sigma 274739), 3. Diatomaceous Earth (SWde, Sigma D5509), 4. Cellulose (SWcel, Sigma S5504), 5. Chitin (SWchi, Sigma C7170) autoclaved and dissolved in $20 \mathrm{~mL}$. Low levels of pyruvate $(0.1 \%$ final concentration) and yeast extract $(0.01 \%$ final concentration) were also added to each sample, which were then incubated for 2 weeks at $15^{\circ} \mathrm{C}$ with $12 \mathrm{~h} \mathrm{light/dark} \mathrm{cycles} \mathrm{with} \mathrm{constant} \mathrm{stirring.} \mathrm{In} \mathrm{order} \mathrm{to}$ collect particle-associated microorganism after these 2 weeks of incubation each particle-enriched culture $(500 \mathrm{ml})$ was filtered through a $20 \mu \mathrm{m}$ filter, allowing sea water to flow through and retaining only the particles and any bacteria attached to them. This was followed by 3 washes, in which $300 \mathrm{~mL}$ of sterile sea water was added to the particles. It should be noted that the control sample (SW), which did not contain any particles, was not subjected to any treatment until the end of the 5 weeks incubation. The whole sample was spun at 13,500 rpm for $30 \mathrm{~min}$ to pellet the microbial cells. A schematic representation of the enrichments experimental design is shown in Figure S1.

\section{DNA Extractions and Metagenomic Sequencing}

Community DNA extractions were performed for two FL and PA fractions (summer and winter), using the 0.22 and $5 \mu \mathrm{m}$ filters, respectively. For the PE metagenomes, community DNA extractions were performed using either pelleted cells (SW) or rinsed and filtered particles (SWsnd, SWde, SWcel, and SWchi). In all cases, $5 \mathrm{~mL}$ of lysis buffer (40 mM EDTA, $50 \mathrm{mM}$ Tris $\mathrm{pH}=8.3$, and $0.75 \mathrm{M}$ Sucrose) were added to the biomass (filters for FL and PA, cell pellet for SW and $5 \mathrm{~mL}$ of particles in all other samples) and incubated at $37^{\circ} \mathrm{C}$ for $45 \mathrm{~min}$ with $50 \mu \mathrm{L}$ Lysozyme $[100 \mathrm{mg} / \mathrm{mL}]$. Nucleic acids were extracted with phenol/chloroform/isoamyl alcohol and chloroform/isoamyl alcohol and DNA integrity was checked by agarose gel electrophoresis. The metagenomes were sequenced using the Illumina Hi-Seq 2000 (100-bp pairedend read) sequencing platform by GATC Biotech (Konstanz, Germany). The IDBA-UD de novo assembler (Peng et al., 2012) was used to assemble all datasets using a Kmer range $(K$ $=70-100$, steps of 10$)$. Gene predictions on the assembled contigs were performed using Prodigal (Hyatt et al., 2010), and tRNAs were predicted using tRNAscan-SE (Lowe and Eddy, 1997). Ribosomal rRNA genes were identified using ssualign (Nawrocki, 2009) and meta_rna (Huang et al., 2009). Predicted proteins extracted from the contigs were assigned functional annotation using USEARCH (Edgar, 2010) against COG (Tatusov et al., 2001), TIGRFAM (Haft et al., 2001) and NCBI nr databases. TIGRFAM and COG assignments were made using an evalue of $<1 \mathrm{e}-5$, and $>50 \%$ query coverage and $>30 \%$ identity for non-redundant NCBI. Particle-associated metagenome were also annotated using the RAST server (Aziz et al., 2008). The completeness and contamination of each reconstructed genome were estimated using CheckM with default settings (Parks et al., 2015). The completeness was estimated by identification of lineage-specific single-copy marker genes and contamination was evaluated from the number of multi-copy marker genes.

\section{Phylogenetic and Functional Classifications}

Phylogenetic classification of the metagenomic data was performed using three different methods. In the first, ribosomal RNA (rRNA) sequences were used as queries for BLASTN searches against the entire RDP database and classified into a high level taxon if the sequence identity was $\geq 80 \%$ and the alignment length was $\geq 90$ bp. For the second method MetaPhlAn version (v2.0) was used to study the microbial community (Segata et al., 2012) from the total reads. This program maps reads to a set of selected marker sequences that are unique for each organism in the database. In addition, we used only the annotation of large contigs ( $>10 \mathrm{~Kb}$ ) to make class-level and organism-level assignments. Genome trees were made for phylogenetic comparisons using conserved protein sequences among the microorganisms used in a given comparison. Conserved proteins were identified for each genome and the reference genomes using the COG database (Tatusov et al., 2001). COG assignments were made using the HMMER software package version 2.0 (Finn et al., 2011) with an evalue of $<1 \mathrm{e}-5,>80 \%$ query coverage and $>30 \%$ identity. The common conserved proteins were identified and concatenated. The resulting concatenated protein sequences were then aligned using Kalign (Lassmann and Sonnhammer, 2005), trimmed using trimAL (Capella-Gutiérrez et al., 2009). A maximum likelihood tree was constructed using FastTree2 (Price et al., 2010) using a JTT+CAT model, a gamma approximation and with 100 bootstrap replicates.

\section{Recruitment Analysis}

The assembled contig groups for each of our PE metagenomes, which were identified at the genus/species level, were compared to a number of metagenomic datasets from seawater to evaluate the coverage of each contig group in natural samples. Recruitments were performed using BLASTN (Altschul et al., 1997) and a hit was considered only when it was at least 50 nucleotides long, with a \%identity of $>95 \%$ and with an $e$-value $\leq 1 \mathrm{e}-5$. The positive results were calculated and reported as reads per $\mathrm{Kb}$ per $\mathrm{Gb}$ of data (RPKG) that provide a normalized value comparable across various metagenomes (Mizuno et al., 2013).

\section{Data Availability}

The metagenomic data and assembled genome sequences have been submitted to NCBI under the Bioproject identifier PRJNA257723 for the PA and FL metagenomes and PRJNA318731 for PE metagenomes. FL-Summer and PA-Summer metagenomes have been submitted as MedDCM-SEP2013 (BioSample SAMN02954150) and MedDCM-SEP2013-LF (BioSample SAMN02954152), respectively. FL-Winter and PA-Winter metagenomes have been submitted as MedWinter-JAN2015-20m (BioSample SAMN04874669) and MedWinter-JAN2015-20m-LF (BioSample SAMN04880534), respectively. 


\section{RESULTS AND DISCUSSION}

\section{Comparison of Free-Living (FL) and Particle-Associated (PA) Metagenomes}

In order to investigate the differences between microbial communities associated with the FL and PA fractions of pelagic seawater in the Mediterranean Sea, we first used size-based filter fractions to produce metagenomes. We compared four large (one Illumina lane) metagenomes sequenced from either the $0.22-5.0 \mu \mathrm{m}$ (FL) or the $5.0-20.0 \mu \mathrm{m}$ (PA) filter fractions of two separate samples. One sample was taken during the summer from the deep chlorophyll maximum (DCM), a zone of maximal phytoplankton concentration that generally occurs below the thermocline. In tropical waters this is a permanent feature, whereas in the Mediterranean and other temperate waters the DCM is a seasonal phenomenon (summer and part of spring and autumn). The second sample was collected during the winter mixing that occurs in January. However, the [Chl a] concentration $\left(\mathrm{mg} \mathrm{m}^{-3}\right)$ was only slightly higher during the summer sampling (0.92) compared to the winter sampling (0.75) but similar to the average [Chl $a$ ] observed at the DCM depth in different points in the southwestern Mediterranean Sea that reaches $0.88 \mathrm{mg} \mathrm{m}^{-3}$ (Lavigne et al., 2015). Approximately, 12 $\mathrm{Gb}$ of raw sequence were retrieved from each data set (Table S1). Figure S2 shows the GC\% distribution plot of the individual reads in the data sets. Both FL samples and the PA from 2014 clearly show a peak at $\mathrm{ca}$. 34\% GC consistent with the GC content of the dominant microbe based on rRNA reads, $\mathrm{Ca}$. Pelagibacter (\%GC ca. 30). Interestingly, however, the summer PA fraction had a bimodal GC distribution (Figure S2) with maxima at 39 and $50 \%$. Both PA metagenomes revealed much higher percentages of eukaryotic and viral sequences and lower percentages of bacteria and archaea based on both rRNA analysis and the MetaPhlAn analysis from the total reads (Figure S3). The ratio of eukaryotic to bacterial rRNA (18S:16S) was (0.63 and 0.32) for PA summer and winter respectively and $(0.036$ and 0.063$)$ for FL. Viral DNA was similarly overrepresented in PA regardless of the season (Figure S3). Also the percentage of sequences with predicted proteins of known function was much lower in PA than in FL metagenomes (ca.16 and ca.40\% respectively) independent of the season. These results are similar with previous observations in particles enriched metagenomes (Ganesh et al., 2014; Dupont et al., 2015; Fontanez et al., 2015) and can be largely attributed to the presence of more eukaryotic DNA in the PA fraction. The presence of phage genomes in metagenomes is due to the abundance in the population of cells undergoing the lytic cycle. During this phase a large amount of phage DNA accumulated within the cell and is retrieved with the cellular fraction (Mizuno et al., 2013). Therefore, higher relative abundance of phage reads in the particulate fraction can be interpreted as a reflection of higher infection rate in this cellular fraction (Allen et al., 2012; Ganesh et al., 2014; Dupont et al., 2015).

Based on rRNA data, at the level of major phylogenetic clades, the community structure of all four metagenomes was remarkably well conserved (Figure 1). In order to better characterize the bacterial community associated to the PA, we examined 16S rRNA reads that were unique to the PA metagenomes by removing sequences that exhibited $>95 \%$ identity for over $90 \mathrm{bp}$ to any sequence in the FL metagenomes. Around $10 \%$ of the $16 \mathrm{~S}$ rRNA sequences $(11.1 \%$ for summer PA and $8.7 \%$ for winter PA) were found to be unique to the PA fraction (Figure 1, Table S2). The most abundant groups among the total PA reads were Proteobacteria (Alpha and Gamma), Bacteroidetes and Cyanobacteria. Whereas, after the subtraction, Proteobacteria (Alpha, Delta, and Gamma), Bacteroidetes (Flavobacteria), and Planctomycetes (Planctomycetia) were significantly overrepresented, providing additional support that certain microorganisms within these classes are unique to the PA community (Figure 1) and consistent with several previous studies of particle-associated bacteria (DeLong et al., 1993; Allen et al., 2012; Ganesh et al., 2014). The percentage of Planctomycetia increased from less than $1 \%$ in the individual metagenomes to more than $7 \%$ in the subtraction in both comparisons (summer and winter), while the same is true for the Verrucomicrobia class Opitutae in the winter sample. Previous studies have demonstrated that marine bacteria belonging to the phylum Planctomycetes are more abundant in sinking marine aggregates (DeLong et al., 1993), in the biofilm community living on the surface of different marine species (Gray and Herwig, 1996; Longford et al., 2007; Bengtsson and Øvreås, 2010) and in particle-associated communities (Allgaier and Grossart, 2006). This data also revealed that the Cyanobacteria present in both fractions represented the same groups. Clogging of the PA filter could have been the reason for the retention of high amount of Cyanobacteria in the PA fraction (Crespo et al., 2013), another explanation is that mucous aggregates of cyanobacteria, previously demonstrated (Deng et al., 2015), hindered their passage through the $5 \mu \mathrm{m}$ filter. Notably, unclassified bacteria were among the largest groups of unique rRNA reads (Figure 1, Table S2), even at higher taxonomic levels, suggesting that a significant portion of microorganisms specific to the PA fraction have yet to be characterized.

We performed de novo contig assembly using the complete set of raw reads to further analyze these data sets (Table S1). As is expected when there are large numbers of eukaryotic sequences and significant differences in GC content, we were unable to assemble large contigs from the PA summer metagenome. Nonetheless, we were able to assemble and compare the other three metagenomes. After assembly, contigs can be classified much more reliably using large genomic fragments (minimum $10 \mathrm{~Kb}$ lengths). However, it is important to emphasize that microbes are not assembled proportionally to their abundance, i.e., assembly is only a rough estimate of the relative abundance. Assembled contigs were assigned to each class based on the majority of genes giving highest similarities to genes in genomes present in NCBI nr database (Figure 2). Alphaproteobateria, Gammaproteobacteria, and Cyanobacteria were the most abundant groups in both fractions, consistent with the previously described rRNA classifications. A very remarkable difference found was the proportion of members of Archaea that increased from 5 to $18 \%$ (summer and winter $\mathrm{FL}$ ) to $32 \%$ in the winter $\mathrm{PA}$ metagenome. In both, the FL and PA fractions Euryarchaeota, the majority of which were Group II Euryarchaeota, were the dominant class 


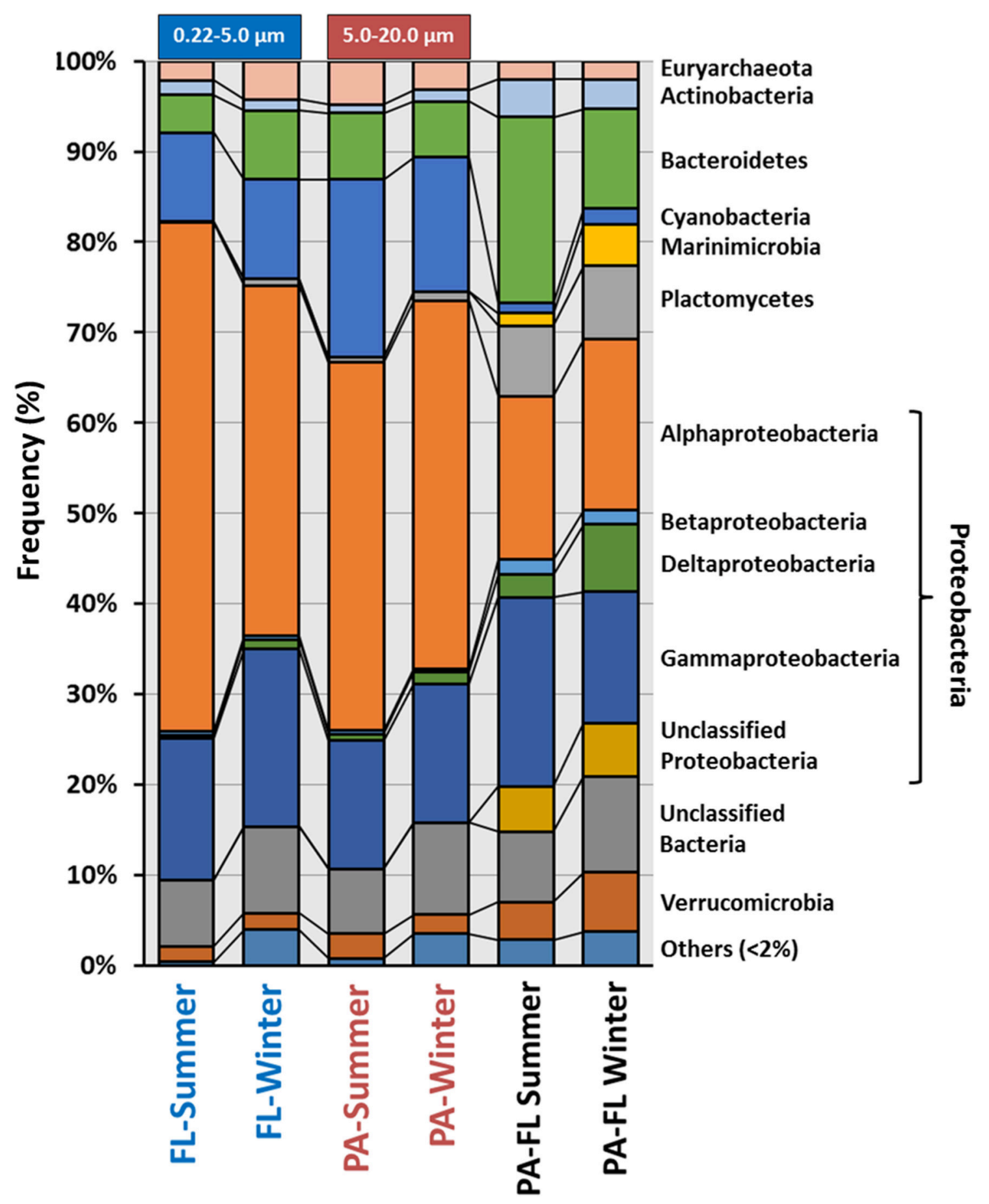

FIGURE 1 | Class-level composition of the domain Bacteria and Archaea based on rRNA (raw reads) among the four individual metagenomes and the subtractive bacterial community associated to the PA metagenomes. For the subtraction, we used prokaryotic rRNA sequences that were found to be unique (i.e., meaning they exhibited >95\% identity for over 90 bp to any sequence in the FL metagenomes) to the PA fraction. Free-living (FL); Particulate-associated (PA).

over Thaumarchaeota. Actinobacteria were more abundant in both FL fractions, reaching up to $10 \%$, compared to the assembled PA contigs, in which it was practically absent (Figure 2). The summer FL metagenome contained mostly $\mathrm{Ca}$.
Actinobacteria minuta (Ghai et al., 2013) and MedAcidi-G2A (Mizuno et al., 2015), while MedAcidi-G3 was predominant in the winter FL. This data suggests that these Actinobacteria do not form aggregates and can pass the $5 \mu \mathrm{m}$ filter. The 


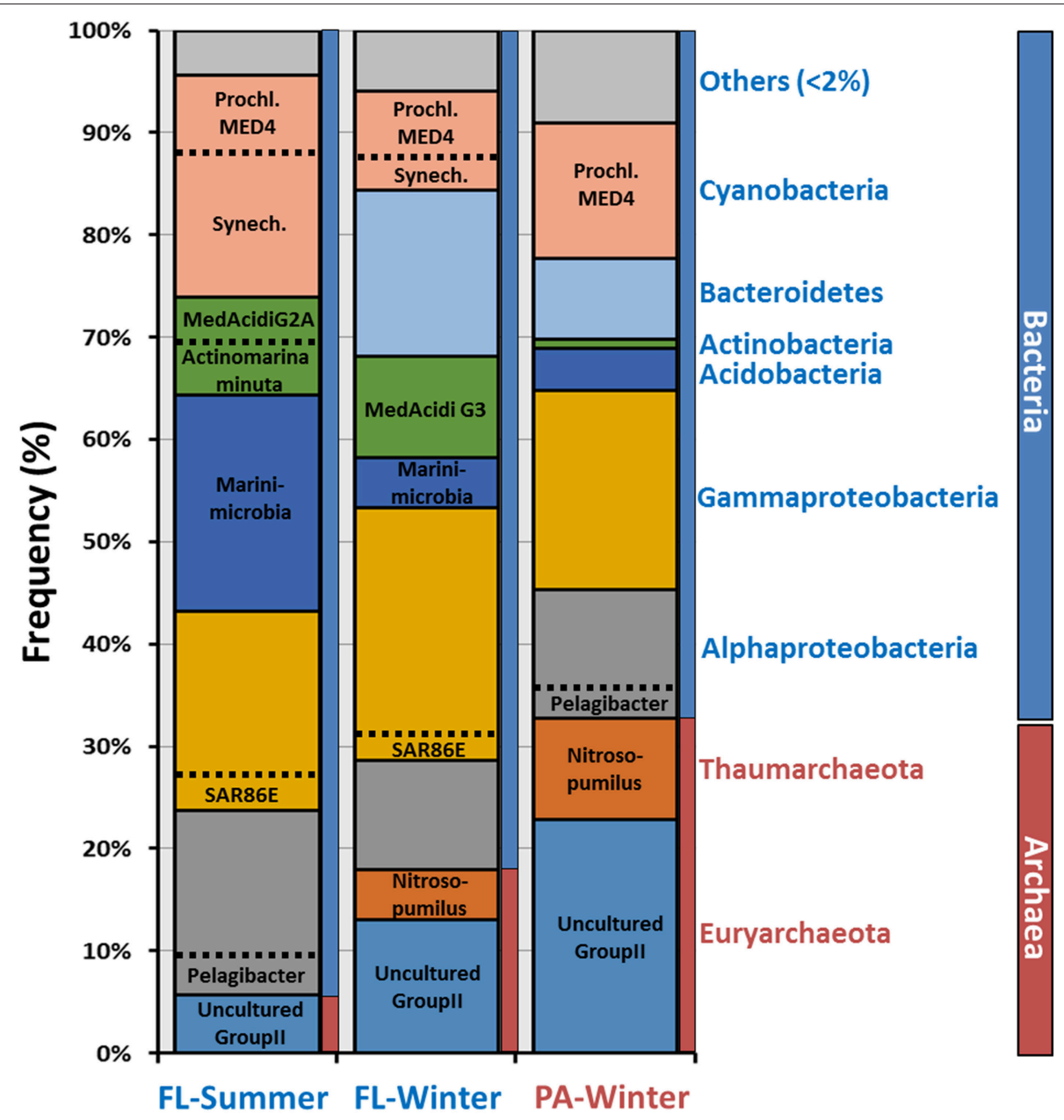

FIGURE 2 | Class-level phylogenetic classification of annotated contigs $>\mathbf{1 0} \mathbf{~ K b}$. When it was possible, contigs were associated at the genus-level based on annotation. Prochl, Prochlorococcus; Synech, Synechococcus.

majority of Gammaproteobacteria contigs were unclassified (Figure 3), suggesting the possibility of novel planktonic lineages. Cyanobacteria were more abundant in the summer FL fraction (21\%) than in the winter FL and PA fraction (10 and $13 \%$, respectively). Moreover, the proportion of the two most abundant Cyanobacteria genera (Prochlorococcus and Synechococcus) varied among the three different metagenomes (Figure 2). The winter PA was particularly enriched in the highlight-adapted Prochlorococcus MED4. Although, this was also the most abundant in the other winter sample, Synechococcus represented $30 \%$ of the Cyanobacteria assembled sequences in the FL fraction. The percentage of Synechococcus increased to $70 \%$ in the summer FL.

\section{Particle Enrichments (PE) and Metagenomic Sequencing}

After comparing the FL and PA metagenomes derived from different size fractions, we designed another approximation to analyze the community that live associated to particles using enrichment metagenomics (Figure S1). In these experiments Mediterranean seawater was enriched with a specific particulate matter. After 2 weeks of growth under ecologically relevant conditions, microbial DNA was extracted for metagenomic sequencing (Table S3). Four types of particulate material were used. Two of them, sand (SWsnd) and diatomaceous earth (SWde) are both inorganic forms of silicates. Sand particles are larger with flat surfaces, while SWde are smaller and with perforated surfaces (larger surface/volume ratios). The other two particulate materials used, cellulose (SWcel) and chitin (SWchi), are organic polymers that could act as an additional carbon source to the pyruvate supplied, and both are abundant in marine POM (Figure S4).

Comparison among the assembled contigs $(>1 \mathrm{~kb})$ of the PE metagenomes based on principal component analysis (PCA) related to taxonomic and functional classifications reveal three clusters. The sequences from the control (SW), devoid of added particles, and the SWcel enrichment share a higher level of similarity in both comparisons, while the two with inorganic forms of silicates (SW snd and SWde) grouped together only 
A

Taxonomic Classifications

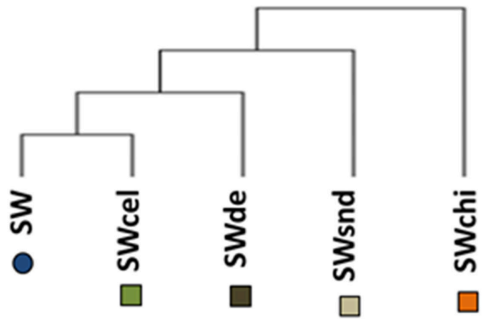

B

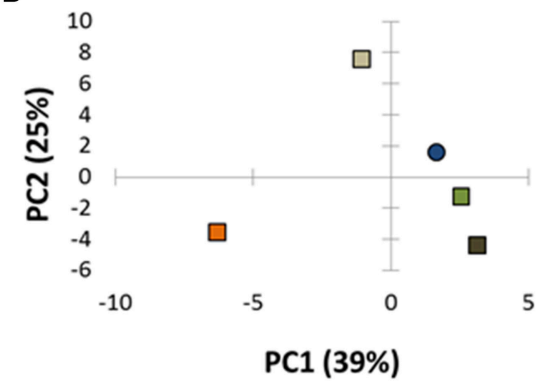

rRNA sequences

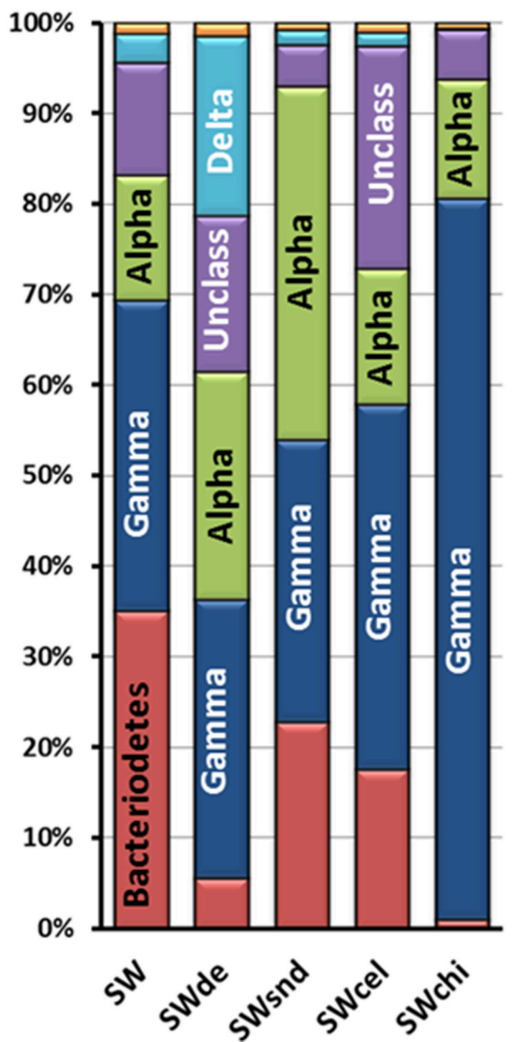

Functional Classifications
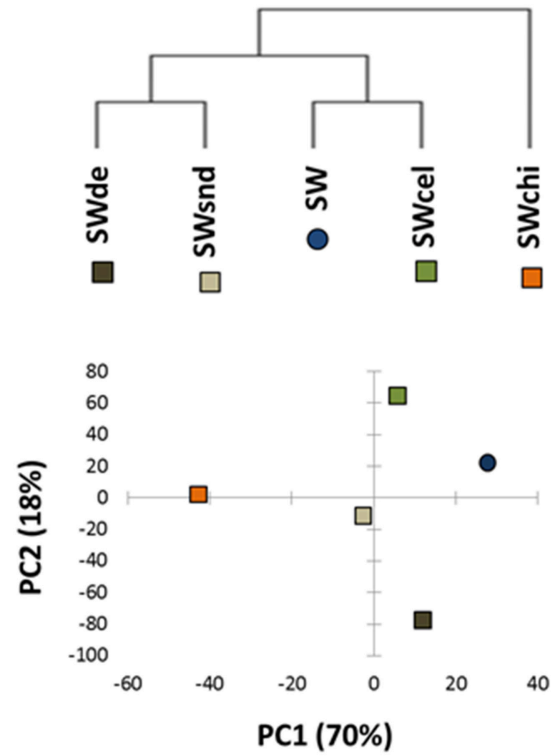

Contigs $>10 \mathrm{~Kb}$

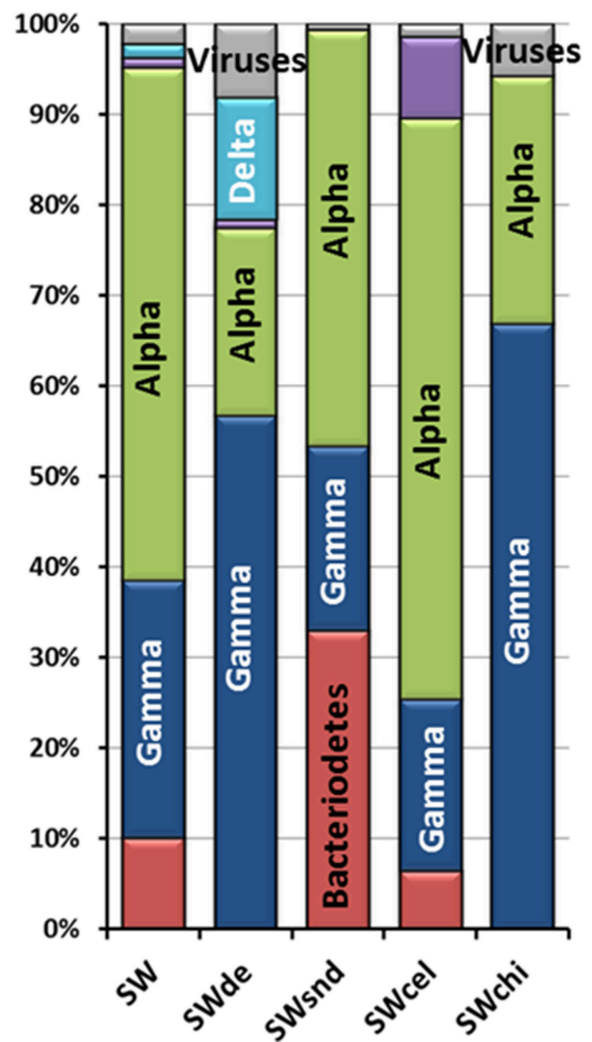

FIGURE 3 | Analyses of the particle-enrichment metagenomes. (A) Metagenomes tree, (B) principle component analysis (PCA) analysis related to taxonomic and functional classifications, and (C) phylogenetic classification of both rRNA sequences (>100 bp) from the raw metagenomic reads and annotated contigs $>10$ Kb for the SW, (sea water-control); SWsnd, (Sand); SWde, (Diatomaceous Earth); SWcel, (Cellulose); and SWchi, (Chitin) metagenomes. 
at the functional level. The most distinct metagenome was the chitin enriched (SWchi), which always appeared as an outgroup (Figures 3A,B). We used the phylogenetic classification of both annotated contigs $>10 \mathrm{~Kb}$ and rRNA sequences (>100 bp) from the raw metagenomic reads (Figure $3 \mathrm{C}$ ) to identify the dominant microorganisms in each metagenome. Even at the class level, we observed distinct community structures with both approaches. Members of the class Alphaproteobacteria (mostly Roseobacter spp) were the best assembled microbes. SWchi and SWde enrichments were dominated by Gammaproteobacteria and a small proportion of Viruses contigs respectively. Using the larger assembled contigs $(>10 \mathrm{~Kb})$ for classification also allowed us to identify a number of organisms at the genus/species level, providing further evidence of variability between the particle enrichments. We have determined a total of 20 groups of contigs associated to a specific microbe (Table 1) from these enrichments, although only eight of these groups of contigs obtained were partial to near complete (50-100\%) and $<4 \%$ contamination (see Materials and Methods, Table S4). These groups of contigs revealed a number of previously undescribed species within the Rhizobiales, Rhodobacteraceae, Flavobacteraceae, Bdellovibrionaceae, and Vibrionaceae families based on average nucleotide identity (ANI) comparisons between the assembled contigs and their corresponding most closely related cultured relative (Table $\mathbf{1}$ ).

\section{The Same but Different, Roseobacter}

All of the PE metagenomes contained Rhodobacteraceae contigs that were identified as Roseobacter sp. (Table 1). All vs. all ANI comparisons that were performed using the four Roseobacter contig groups and the closest sequenced relative, Roseobacter sp. MED193, in order to determine the phylogenetic relationship between the enriched Roseobacter spp. (Figure S5A). The results indicate that they represent three distinct species with only the SWde and SWcel sharing a high enough ANI to be considered as the same species as the cultured relative Roseobacter sp. MED193 (SWde, 98\% ANI with 88\% coverage; SWcel, 97.6\% ANI with $73 \%$ coverage). Furthermore, SWsnd and SWchi contigs can be considered borderline within the same species sharing an ANI of 94.8\% (58\% coverage), while the SW Roseobacter spp represent a new Roseobacter species (Figure S5A). Phylogenetic analysis using 42 conserved proteins provided additional evidence supporting the four distinct Rhodobacteraceae groups (Figure $\mathrm{S} 5 \mathrm{~B})$. The results indicate that particle size was the most relevant parameter to determine the overall community similarity i.e., similarly sized particles had similar communities regardless of the particle composition. For example, SWsnd and SWchi (large particles) had similar Roseobacter species attached although they are chemically very different.

\section{Specific Microorganism from Particle Enrichments Metagenomes}

One group of 28 contigs from the SW control were identified as a Rhizobium sp. (Table 1), with the most closely related species being the newly described Mediterranean isolate, Pseudorhizobium pelagicum R1-200B4 ${ }^{\mathrm{T}}$ (Kimes et al., 2015). This microbe represents the first marine member of the family
Rhizobiaceae. The alignment of the SW-Rhizobium contigs with the genome sequence of this isolate (accession NCBI number NZ_JOKI00000000.1) shows both sequence similarity and synteny, while the genome recruitment of Pseudorhizobium pelagicum $\mathrm{R} 1-200 \mathrm{~B} 4^{\mathrm{T}}$ with the SW metagenomic dataset reveals full coverage of the genome (Figure S6A). A conserved protein tree provides further evidence that the SW contigs form a cluster with both Pseudorhizobium pelagicum R1-200B4 ${ }^{\mathrm{T}}$ and Rhizobium sp. NT26 (Figure S6B). The second group of contigs detected in SW, a Flavobacteraceae group, was identified as a Lacinutrix sp., most closely resembling Lacinutrix sp 5H-3-74. This member of the family Flavobacteriaceae was isolated from subseafloor sediments using a mixture of cellulose, xylan, and chitin as the sole carbon sources in enrichment cultures (Klippel et al., 2011). The ANI (93\% with 83\% coverage) between the SW contigs and Lacinutrix sp 5H-3-7-4 genome indicated that, although potentially a different species, both are closely related. Moreover, the genome completeness based on CheckM was $99.34 \%$ with $0 \%$ of contaminations and this microbe recruited up to $34 \%$ of all reads belonging to the SW metagenome (Table S4). Alignment between contigs and the reference genome indicated that synteny was very well preserved except for the presence of some genomic islands. Although, most are associated with cell wall synthesis, we have found several glycoside hydrolases, such as laminarinase and alpha-amylase that could facilitate the use of several natural substrates as sources of carbon and energy. The SWcel metagenome contained only one group of Flavobacteraceae contigs $(n=22)$. However, unlike the SW contigs the SWcel were classified as a Flavobacteria sp. (Table 1). The most closely related microorganism identified, Flavobacterium beibuense $\mathrm{F} 44-8^{\mathrm{T}}$, clearly represented a different species ( $72 \%$ ANI with $44 \%$ coverage). This microbe was isolated from a crude-oil-degrading consortium, enriched from marine sediment (Fu et al., 2011). In total, the 23 Flavobacteria contigs, ranging between 136 and $12 \mathrm{~Kb}$, represented more than one half $(2.5 \mathrm{Mb})$ of the expected genome size $(3.8 \mathrm{Mb})$. The genome was estimated to be $88.7 \%$ complete and with minimal contamination (Table S4). Recruitment of identical reads with the reference metagenome (SWcel) showed that $10.7 \%$ of the reads belonged to this Flavobacterium beibuense-like contigs. Their GC content was $33.8 \%$, which is lower than that of Flavobacterium beibuense $\mathrm{F} 44-8^{\mathrm{T}}(37.7 \%)$. The contigs contain a cluster of $5 \mathrm{crt}$ genes involved in carotenoid biosynthesis and genes associated with gliding motility, all typical characteristics of members of the class Flavobacteriia. No cellulases could be detected neither in the genome nor in the reference genome and it is possible that this specific microbe is only using the particles as physical support. Interestingly, we have found a $30 \mathrm{~Kb}$ putative prophage inserted in the genome with a significant gene similarity and synteny to another prophage inserted in the same position in a soil Bacteroidetes, Flavobacterium sp. CF136, isolated from the Populus deltoids rhizosphere (Brown et al., 2012). Based on ANI identity both strains represented different species (70\% ANI with only $24 \%$ coverage).

Diatomaceous earth is made up of silicate remains of fossilized diatoms and (like in the case of sand) provides no organic carbon. The SWde metagenome contained a group 


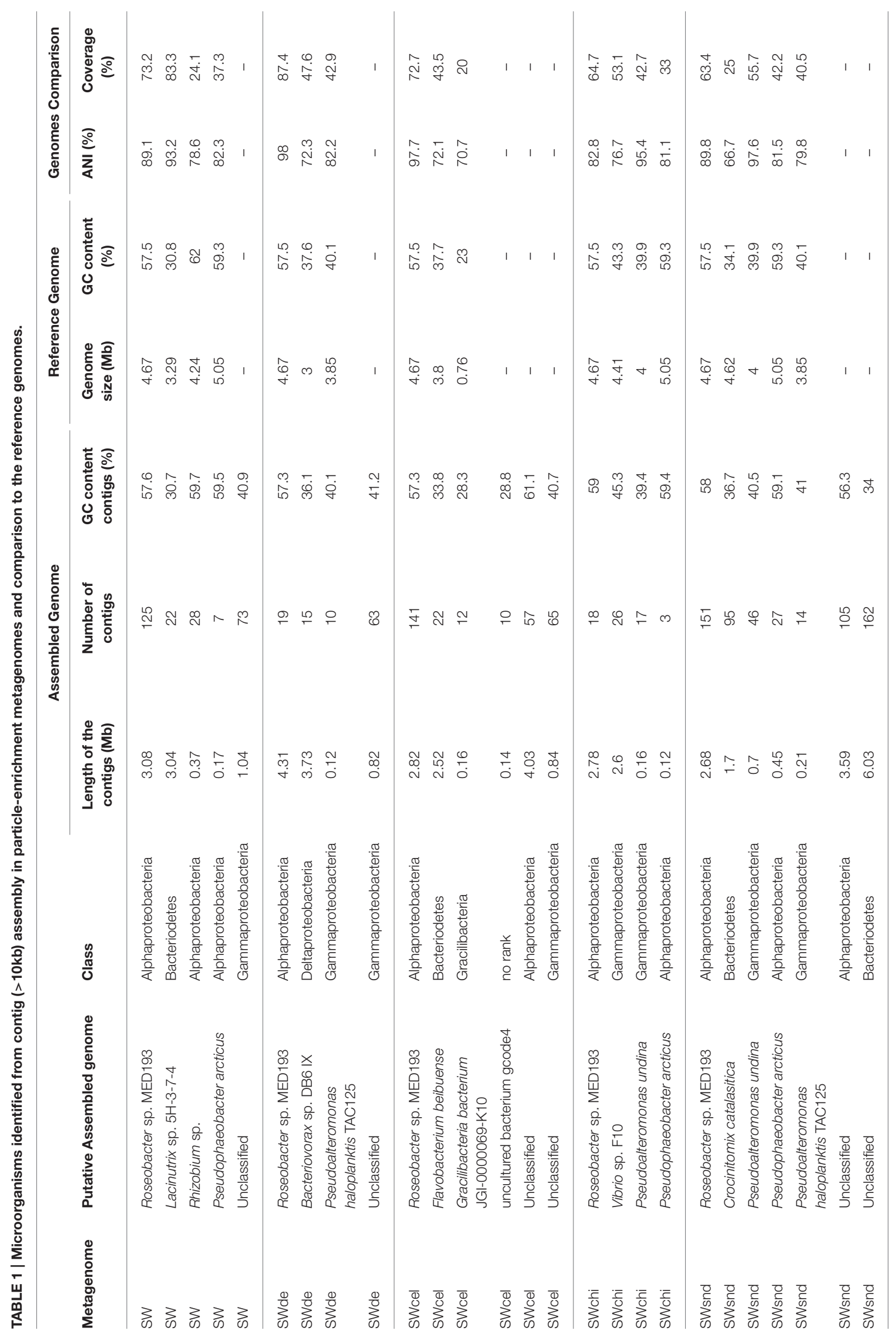


of Deltaproteobacteria contigs identified as being most closely related to Bacteriovorax sp. DB6. Bacteriovorax is a genus of predatory bacteria related to Bdellovibrio that prey upon Gramnegative bacteria by invading their periplasmic space. They also exhibit an extracellular free-living phase in their lifecycle, during which they establish natural biofilms to deal with harsh environmental conditions (Williams et al., 2009). The 15 contigs in this group provided nearly complete genome coverage (3.73 $\mathrm{Mb}$ ) since the average size for the four available genomes of Bacteriovorax spp is $3.31 \mathrm{Mb}$. However, the percentage of completeness showed that we only have $54.46 \%$ (Table S4) of the genome. The lack of members of the same genus in the databases makes the program determine marker genes only at the kingdom level. Although, the GC content of the SWde contigs (36.1\%) and Bacteriovorax sp. DB6 genome (37.6\%) are similar, ANI (72\% with $48 \%$ coverage) indicated that they were clearly separate species. Phylogenetic analysis (64 conserved proteins) supported that the SWde Bacteriovorax contigs were grouped together with Bacteriovorax spp. cluster IX (group based on 16S rRNA, Chen et al., 2015) in the Bdellovibrionaceae branch of Deltaproteobacteria (Figure S7A). The presence of Bacteriovorax suggests that under specific environmental conditions the predation exhibited by this microbe could be an important factor in shaping the community composition. Although, it has been suggested that Bacteriovorax showed preferential predation toward Vibrio parahaemolyticus in seawater (Richards et al., 2013), neither assembled contigs nor raw reads obtained in the SWde metagenome could be assigned to Vibrio. Annotation of these genomes gave a very high rate of hypothetical proteins (annotated genes account only for $\sim 32 \%$ ) which complicates comparisons among them.

A group of Gammaproteobacteria contigs resembled the not yet formally described species Vibrio sp. F10 and was observed only in the SWchi metagenome. Vibrio sp. F10 appears to be a highly specific zooplankton saprophyte (Preheim et al., 2011). The ANI between this group of contigs ( 26 adding up to $2.6 \mathrm{Mb}$ ) and the Vibrio sp. F10 draft genome (77\% with 53\% coverage) indicated that the enriched microbe was a different species, although with a similar GC content (ca. 45\%). The metagenomic contigs alignment to Vibrio sp. F10 also indicated low synteny. A phylogenetic tree using 86 conserved proteins supports the grouping of the SWchi enriched vibrio contigs with Vibrio sp. F10, which forms a distinct cluster from other characterized vibrios (Figure S7B). Notably, the Vibrio contigs from the SWchi metagenome contain two chitinases supporting their association with this substrate.

The chitin particles were colonized by Vibrio species related to the genome described as Vibrio sp. F10, while the cellulose particles were colonized by members of the Flavobacterium genus. Slightly more surprising, however, was the development of distinct communities associated with the particles providing no additional carbon sources. Diatomaceous earth, for example, is a material made up of the silicate remains of fossilized diatoms and provides no organic carbon, similar to sand. Nonetheless, we observed very different communities adhering to both silicate-based materials. In this case the physical structure of diatomaceous earth, which is much more convoluted and full of crevices, could be the major factor. Additional experiments with biological replicates, not available at this point, would strengthen the value of the results. Another important factor which might contribute to the differences in community composition on the different particles could be also the differences in the adsorption of DOM on these particles, depending on the surface charge and the surface structure. This is critical in primary film formation which in turn, determines the microbes colonizing the surface. Also quite remarkable was that the most fully assembled microbe in this enrichment was a predatory bacterium. These micropredators have been isolated in different aquatic systems (marine, estuaries, and freshwater; Jurkevitch, 2007) and takes advantage of small perturbations in the environment that allow rapid growth of prey organisms (Williams et al., 2016). However, although the predatory life cycle has been well studied (Rendulic et al., 2004) little is known about the extracellular free-living phase because most of them are unable to grow in the absence of prey. It is possible that Bacteriovorax takes advantage of the complex surface structure to find prey more easily.

\section{New Groups of Marine Bacteria}

Within the classification of long contigs ( $>10 \mathrm{~kb}$; Table $\mathbf{1}$ ), we found some representing unclassified microbes in which the majority of genes gave best hits to different organisms of the same class. We have used a combination of GC\%, coverage, and principal component analysis of tetranucleotide frequencies in order to bin the unknown contigs into groups as was previously described (Mizuno et al., 2015). Table 2 summarizes some of the statistics of these new groups of Alphaproteobacteria, Bacteroidetes, and Gammaproteobacteria found in the PE metagenomes. Every group seems to be different in each metagenome except for the contigs belonging to Gammaproteobacteria for which the same group appeared to be present in all the metagenomes.

We have used orthologous markers to estimate the completeness of the recovered genomes. These markers indicated that the range of genome recovery was higher than 68.5\% using Albertsen estimation (Albertsen et al., 2013) and $80 \%$ for Raes (Raes et al., 2007) for all the assembled genomes. Unfortunately, only in one of these groups we found a contig containing a partial 16S rRNA gene (Gammaproteobacteria MedPE). Reconstructed phylogeny of the 16S rRNA showed that our groups of contigs were related to other uncultured microbes being most similar to a bacterium associated with the sponge Petrosia ficiformis collected along the North Western Mediterranean coast named Gamma proteobacterium MOLA 543. In order to make a more robust phylogenetic affiliation and be able to place the groups without any 16S rRNA among the contigs we also used a concatenate of conserved proteins between these and several reference genomes chosen based on the majority of genes in the contigs giving highest similarities to genes in databases. Between the two groups of Alphaproteobacteria the SWcel group clustered together in the tree with members of the family Rhodobacteraceae (Figure S8A). Highest similarity based on ANI (77\%) was with Silicibacter sp. strain TM1040 an isolate obtained from a culture of the dinoflagellate Pfiesteria piscicida. However, SWsnd contigs bin 
TABLE 2 | Summary statistics of the reconstructed genomes obtained in the enrichment metagenomes.

\begin{tabular}{|c|c|c|c|c|c|c|}
\hline Cluster name & Number of contigs & GC (\%) & Total length (bp) & $\begin{array}{l}\text { Longest contig } \\
\text { size (bp) }\end{array}$ & $\begin{array}{c}\% \text { completeness } \\
\text { (Raes) }\end{array}$ & $\begin{array}{c}\% \text { completeness } \\
\text { (Albertsen) }\end{array}$ \\
\hline Alphaproteobacteria MedPE-SWcel & 54 & 61.4 & $4,076,435$ & 311,167 & 100 & 83.8 \\
\hline Alphaproteobacteria MedPE-SWchi & 100 & 56.6 & $3,548,614$ & 120,524 & 80 & 68.5 \\
\hline Bacteroidetes MedPE-SWsnd-G1 & 60 & 33.4 & $2,702,127$ & 196,890 & 85.7 & 75.7 \\
\hline Bacteroidetes MedPE-SWsnd-G2 & 19 & 34.3 & $3,035,083$ & 480,119 & 100 & 83.8 \\
\hline
\end{tabular}

appeared as an outgroup of the tree probably forming a new genus. The assembled contigs of both new Alphaproteobacteria were submitted to the RAST annotation server for subsystem classification (Aziz et al., 2008). SWcel Alphaproteobacteria revealed an increase number in genes involved in membrane transport such as $\mathrm{ABC}$ transporters, iron acquisition and carbohydrates including mono, polysaccharide utilization and one-carbon metabolism in comparison to the SWsnd. However, the main difference in the Alphaproteobacteria SWsnd genome was the presence of a nitrous oxide reductase cluster of genes (nosRZDFYLX) involved in denitrification. Two new groups of Bacteroidetes came from the same metagenome (SWsnd), the resulting trees (Figure S8B) with several reference Bacteroidetes genomes showed that nearest neighbors for the first group were Polaribacter spp and Winogradskyella spp for the second. However, their similarity with the some representative genomes was rather low (ANI $<60 \%$ ), suggesting that they belong to different genera. All these five new groups are estimated to be $>79 \%$ complete with minimal estimated contamination $(<1.8 \%)$ (Table S4). Estimating the percentage of identical reads that match to the corresponding metagenome, these partial genomes accumulated between 3 and $9 \%$ of the reads (Table S4).

\section{New Marine Rhizobiales Phage}

From all the assembled long contigs $(>10 \mathrm{~kb})$ some of them were related to phages. Most affiliated with Vibrio and Pseudoalteromonas phages based on sequence similarities. However, in the SWcel metagenome we have found a 45 $\mathrm{Kb}$ phage with $55.5 \%$ GC content encoding 54 CDSs, of which 18 showed similarities to a group of lytic phages that infect Rhizobium etli isolated from rhizosphere soil of bean plants from agricultural lands in Mexico (Santamaría et al., 2014). Despite the different origin (soil and seawater) synteny between these two phages was well conserved (Figure 4). Based on the large terminase protein of this phage, we built a phylogenetic tree (Figure S9) using the 100 most similar sequences identified in GenBank via BLASTP. The tree shows that, although the SWcel phage grouped together with the Rhizobium phage RHEph02 and RHEph08, it was more similar to a hypothetical protein found in Pseudorhodobacter antarcticus, an Alphaproteobacteria isolated from Antarctic intertidal sandy sediment (Chen et al., 2013). Alignment between the SWcel phage and $P$. antarcticus (Figure 4) revealed that this microbe had a similar and syntenic prophage inserted in a tRNA-Met. Therefore, it is remarkable that this new phage seems to belong to a group that infects a broad range of Alphaproteobacteria that live in habitats as diverse as soil, marine sediment and water column around the world, from agricultural lands in Mexico to the Mediterranean Sea through the Antarctic. Little is known about the movement of viruses among different biomes. There are evidences of some conserved phage-encoded sequences found in multiple different environments (marine, freshwater, soil, sediment, and hypersaline environments; Breitbart et al., 2004; Short and Suttle, 2005) and experiments with viruses from soil, sediment, and freshwater that were able to infect marine microbial communities (Sano et al., 2004) suggesting that viruses are important elements of lateral gene transfer between different compartments of the biosphere.

\section{Recruitment of the Enriched Microorganisms}

To examine the distribution of the novel enriched microorganisms described above we performed fragment recruitment analysis by comparing each contig group to that of numerous metagenomes from a variety of seawater samples (DeLong et al., 2006; Rusch et al., 2007; Coleman and Chisholm, 2010; Eloe et al., 2011; Quaiser et al., 2011; Smedile et al., 2013; Thompson et al., 2013; Wilkins et al., 2013; Mizuno et al., 2015; Sunagawa et al., 2015; Table S5). The resulting RPKG values, which provide an estimated coverage, revealed very low, if any, recruitment of the enriched microorganisms from natural samples. Neither the place of origin (Mediterranean Sea, Baltic Sea, Atlantic, and Pacific Ocean) nor the filter fraction used (ranging between 20 and 0.1 $\mu \mathrm{m})$ had a significant effect in the abundance of the enriched microorganism and their most closely related genomes. Only the genomes related to Pseudoalteromonas species recruited significantly at some stations (TARA 70 South Atlantic Ocean [800 m] and TARA 93 from South Atlantic Ocean [5 and $30 \mathrm{~m}]$ ) from the FL fraction in the TARA metagenomes. The lack of recruitment observed here suggest that particle enrichment, like many other culturing techniques, selects for a small subset of microorganisms that flourish in these specific conditions but do not fare so well in more complex natural environments. These kinds of microbes which exist at nominal levels under natural conditions are part of the denominated "rare biosphere" (Sogin et al., 2006). We have also analyzed the presence of all these novel microorganisms against the 


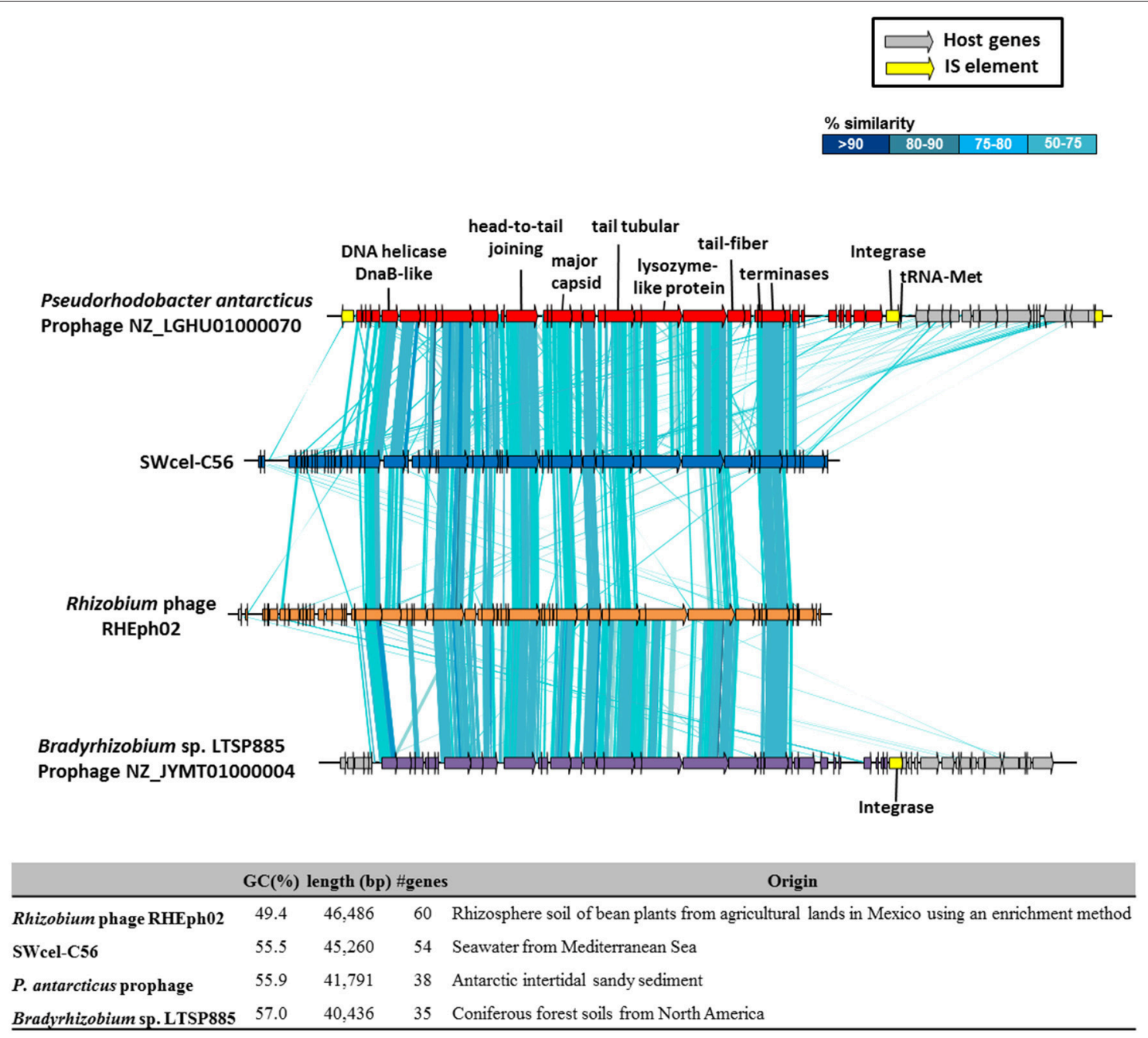

FIGURE 4 | Schematic representation and comparison of the putative phage found in the SWcel enriched metagenome and the closely related phages and prophages based on the sequence of the terminase (Figure S9). The table shows the general features of the genomes.

four PE metagenomes and the control (SW) using not only RPGK (Table S5) but also the percentage of total reads from the metagenome that match to each genome (at 100\% identity; Table S4). The total percentage of metagenomic reads recruited by the genomes assembled here ranged from 17\% (SWchi) to 52\% (SW). Although, far from being diagnostic as to what microbes predominated in the enrichments, our results describe novel genomes that appear in significant numbers under the conditions studied.

\section{CONCLUSIONS}

We have used two metagenomic approaches, direct sequencing of natural samples and sequencing after enrichment, to characterize communities of prokaryotes associated to particles. The direct sequencing of biomass collected on $5 \mu \mathrm{m}$ filters, which in theory should collect eukaryotic cells and only prokaryotic cells forming aggregates or associated to particulate matter, accentuates the difficulties associated with evaluating particle-associated microbiomes. Our data has shown that "bona fide" free living bacteria, such as $\mathrm{Ca}$. Pelagibacter, are collected in the large pore size, likely as a result of clogging that produces a much smaller pore size. In addition, the large amount of eukaryotic DNA collected complicates the analysis of these metagenomes at the level of assembly. Despite these complications, the subtractive analysis, i.e., what is present in PA that is not found in FL, indicated the existence of specific groups of Actinobacteria, Planctomycetes, Flavobacteria, and Proteobacteria that were not present in FL. These microbes appear to be undescribed as of yet, even by metagenomics, and justify the use of alternative approaches that target and retrieve this fraction of the marine microbiome.

The use of culture enrichments has provided genomic data about microbes present in the sample that attach to different particulate materials. This data revealed a remarkable degree 
of specificity, with totally different microbes found in the different enrichments, and very little overlap despite the fact that dissolved organic carbon provided for all the enrichments, namely pyruvate, was the same. Some of the particles provided additional sources of carbon and other nutrients that could dictate some specificity. This was particularly clear in the case of chitin where classic chitinolytic microbes of the genus Vibrio were found to dominate (Hunt et al., 2008). Inorganic material, such as sand or diatomaceous earth, however, also supported very distinct communities, suggesting that additional physical properties influence the development and survival of specific microorganisms. Although, the objective of this analysis was to identify microbes (through the reconstructed genomes) that appear when certain kinds of particles were used, in the future a more in-depth analysis should be performed including both biological replicates and microscopy to check the status of the particles at the end of the experiment. Collectively, our data reveals new and distinct bacteria from the marine microbiome and suggests that particles found in this environment likely play a role in defining the marine microbiome.

\section{AUTHOR CONTRIBUTIONS}

FR, NK designed the project. ML, NK analyzed the data. JH contributed bioinformatics tools. ML, NK, and FR wrote the paper.

\section{ACKNOWLEDGMENTS}

This work was supported by projects MEDIMAX BFPU201348007-P from the Spanish Ministerio de Economía $y$ Competitividad, MaCuMBA Project 311975 of the European Commission FP7 and PROMETEO II/2014/012 Project AQUAMET from the Generalitat Valenciana. JH was supported with a $\mathrm{PhD}$ fellowship from the Spanish Ministerio de Economía y Competitividad.

\section{SUPPLEMENTARY MATERIAL}

The Supplementary Material for this article can be found online at: http://journal.frontiersin.org/article/10.3389/fmicb. 2016.00996

\section{REFERENCES}

Albertsen, M., Hugenholtz, P., Skarshewski, A., Nielsen, K. L., Tyson, G. W., and Nielsen, P. H. (2013). Genome sequences of rare, uncultured bacteria obtained by differential coverage binning of multiple metagenomes. Nat. Biotechnol. 31, 533-538. doi: 10.1038/nbt.2579

Allen, A. E., Allen, L. Z., and Mccrow, J. P. (2013). Lineage specific gene family enrichment at the microscale in marine systems. Curr. Opin. Microbiol. 16, 605-617. doi: 10.1016/j.mib.2013.10.001

Allen, L. Z., Allen, E. E., Badger, J. H., Mccrow, J. P., Paulsen, I. T., Elbourne, L. D., et al. (2012). Influence of nutrients and currents on the genomic composition of microbes across an upwelling mosaic. ISME J. 6, 1403-1414. doi: 10.1038/ismej.2011.201

Allgaier, M., and Grossart, H.-P. (2006). Seasonal dynamics and phylogenetic diversity of free-living and particle-associated bacterial communities in four lakes in northeastern Germany. Aquat. Microbial. Ecol. 45, 115-128. doi: 10.3354/ame045115

Altschul, S. F., Madden, T. L., Schäffer, A. A., Zhang, J., Zhang, Z., Miller, W., et al. (1997). Gapped BLAST and PSI-BLAST: a new generation of protein database search programs. Nucleic Acids Res. 25, 3389-3402. doi: 10.1093/nar/25.17.3389

Azam, F. (1998). Microbial control of oceanic carbon flux: the plot thickens. Science 280, 694-696. doi: 10.1126/science.280.5364.694

Azam, F., and Long, R. A. (2001). Oceanography: Sea snow microcosms. Nature 414, 495-498. doi: 10.1038/35107174

Azam, F., and Malfatti, F. (2007). Microbial structuring of marine ecosystems. Nat. Rev. Microbiol. 5, 782-791. doi: 10.1038/nrmicro1747

Azam, F., and Worden, A. Z. (2004). Microbes, molecules, and marine ecosystems. Science 303, 1622-1624. doi: 10.1126/science.1093892

Aziz, R. K., Bartels, D., Best, A. A., Dejongh, M., Disz, T., Edwards, R. A., et al. (2008). The RAST Server: rapid annotations using subsystems technology. BMC Genomics 9:75. doi: 10.1186/1471-2164-9-75

Bengtsson, M. M., and Øvreås, L. (2010). Planctomycetes dominate biofilms on surfaces of the kelp Laminaria hyperborea. BMC Microbiol. 10:261. doi: 10.1186/1471-2180-10-261

Breitbart, M., Miyake, J. H., and Rohwer, F. (2004). Global distribution of nearly identical phage-encoded DNA sequences. FEMS Microbiol. Lett. 236, 249-256. doi: 10.1111/j.1574-6968.2004.tb09654.x

Brown, S. D., Utturkar, S. M., Klingeman, D. M., Johnson, C. M., Martin, S. L., Land, M. L., et al. (2012). Twenty-One Genome Sequences from Pseudomonas
Species and 19 Genome Sequences from Diverse Bacteria Isolated from the Rhizosphere and Endosphere of Populus deltoides. J. Bacteriol. 194, 5991-5993. doi: 10.1128/JB.01243-12

Capella-Gutiérrez, S., Silla-Martínez, J. M., and Gabaldón, T. (2009). trimAl: a tool for automated alignment trimming in large-scale phylogenetic analyses. Bioinformatics 25, 1972-1973. doi: 10.1093/bioinformatics/ btp 348

Chen, C.-X., Zhang, X.-Y., Liu, C., Yu, Y., Liu, A., Li, G.-W., et al. (2013). Pseudorhodobacter antarcticus sp. nov., isolated from Antarctic intertidal sandy sediment, and emended description of the genus Pseudorhodobacter Uchino et al. 2002 emend. Jung et al. 2012. Int. J. Syst. Evolut. Microbiol. 63, 849-854. doi: 10.1099/ijs.0.042184-0

Chen, H., Brinkac, L. M., Mishra, P., Li, N., Lymperopoulou, D. S., Dickerson, T. L., et al. (2015). Draft genome sequences for the obligate bacterial predators Bacteriovorax spp. of four phylogenetic clusters. Stand. Genomic Sci. 10:11. doi: 10.1186/1944-3277-10-11

Coleman, M. L., and Chisholm, S. W. (2010). Ecosystem-specific selection pressures revealed through comparative population genomics. Proc. Natl. Acad. Sci. 107, 18634-18639. doi: 10.1073/pnas.1009480107

Crespo, B. G., Pommier, T., Fernández-Gómez, B., and Pedrós-Alió, C. (2013). Taxonomic composition of the particle-attached and free-living bacterial assemblages in the Northwest Mediterranean Sea analyzed by pyrosequencing of the 16S rRNA. Microbiol. Open 2, 541-552. doi: 10.1002/mbo3.92

Crump, B. C., Armbrust, E. V., and Baross, J. A. (1999). Phylogenetic analysis of particle-attached and free-living bacterial communities in the Columbia River, its estuary, and the adjacent coastal ocean. Appl. Environ. Microbiol. 65, 3192-3204.

DeLong, E. (2013). Microbial Metagenomics, Metatranscriptomics, and Metaproteomics. San Diego, CA: Academic Press.

DeLong, E. F., Franks, D. G., and Alldredge, A. L. (1993). Phylogenetic diversity of aggregate-attached vs. free-living marine bacterial assemblages. Limnol. Oceanogr. 38, 924-934. doi: 10.4319/lo.1993.38.5.0924

DeLong, E. F., Preston, C. M., Mincer, T., Rich, V., Hallam, S. J., Frigaard, N.-U., et al. (2006). Community genomics among stratified microbial assemblages in the ocean's interior. Science 311, 496-503. doi: 10.1126/science. 1120250

Deng, W., Monks, L., and Neuer, S. (2015). Effects of clay minerals on the aggregation and subsequent settling of marine Synechococcus. Limnol. Oceanogr. 60, 805-816. doi: 10.1002/lno.10059 
Dinsdale, E. A., Edwards, R. A., Hall, D., Angly, F., Breitbart, M., Brulc, J. M., et al. (2008). Functional metagenomic profiling of nine biomes. Nature 452, 629-632. doi: $10.1038 /$ nature 06810

Dupont, C. L., Mccrow, J. P., Valas, R., Moustafa, A., Walworth, N., Goodenough, U., et al. (2015). Genomes and gene expression across light and productivity gradients in eastern subtropical Pacific microbial communities. ISME J. 9, 1076-1092. doi: 10.1038/ismej.2014.198

Edgar, R. C. (2010). Search and clustering orders of magnitude faster than BLAST. Bioinformatics 26, 2460-2461. doi: 10.1093/bioinformatics/btq461

Eloe, E. A., Fadrosh, D. W., Novotny, M., Zeigler Allen, L., Kim, M., Lombardo, M.-J., et al. (2011). Going deeper: metagenome of a hadopelagic microbial community. PLOS ONE 6:e20388. doi: 10.1371/journal.pone.00 20388

Fierer, N., Leff, J. W., Adams, B. J., Nielsen, U. N., Bates, S. T., Lauber, C. L., et al. (2012). Cross-biome metagenomic analyses of soil microbial communities and their functional attributes. Proc. Natl. Acad. Sci. U.S.A. 109, 21390-21395. doi: 10.1073/pnas.1215210110

Finn, R. D., Clements, J., and Eddy, S. R. (2011). HMMER web server: interactive sequence similarity searching. Nucleic Acids Res. 39, W29-W37. doi: 10.1093/nar/gkr367

Fontanez, K. M., Eppley, J. M., Samo, T. J., Karl, D. M., and Delong, E. F. (2015). Microbial community structure and function on sinking particles in the North Pacific Subtropical Gyre. Front. Microbiol. 6, 469. doi: 10.3389/fmicb.2015.00469

Fu, Y., Tang, X., Lai, Q., Zhang, C., Zhong, H., Li, W., et al. (2011). Flavobacterium beibuense sp. nov., isolated from marine sediment. Int. J. Syst. Evolut. Microbiol. 61, 205-209. doi: 10.1099/ijs.0.018846-0

Ganesh, S., Parris, D. J., Delong, E. F., and Stewart, F. J. (2014). Metagenomic analysis of size-fractionated picoplankton in a marine oxygen minimum zone. ISME J. 8, 187-211. doi: 10.1038/ismej.2013.144

Ghai, R., Martin-Cuadrado, A.-B., Molto, A. G., Heredia, I. G., Cabrera, R., Martin, J., et al. (2010). Metagenome of the Mediterranean deep chlorophyll maximum studied by direct and fosmid library 454 pyrosequencing. ISME J. 4, 1154-1166. doi: 10.1038 /ismej.2010.44

Ghai, R., Mizuno, C. M., Picazo, A., Camacho, A., and Rodriguez-Valera, F. (2013). Metagenomics uncovers a new group of low GC and ultra-small marine Actinobacteria. Sci. Rep. 3:2471. doi: 10.1038/srep02471

Giovannoni, S. J., Tripp, H. J., Givan, S., Podar, M., Vergin, K. L., Baptista, D., et al. (2005). Genome streamlining in a cosmopolitan oceanic bacterium. Science 309, 1242-1245. doi: 10.1126/science.1114057

Gray, J. P., and Herwig, R. P. (1996). Phylogenetic analysis of the bacterial communities in marine sediments. Appl. Environ. Microbiol. 62, 4049-4059.

Haft, D. H., Loftus, B. J., Richardson, D. L., Yang, F., Eisen, J. A., Paulsen, I. T., et al. (2001). TIGRFAMs: a protein family resource for the functional identification of proteins. Nucleic Acids Res. 29, 41-43. doi: 10.1093/nar/29.1.41

Huang, Y., Gilna, P., and Li, W. (2009). Identification of ribosomal RNA genes in metagenomic fragments. Bioinformatics 25, 1338-1340. doi: 10.1093/bioinformatics/btp161

Hunt, D. E., Gevers, D., Vahora, N. M., and Polz, M. F. (2008). Conservation of the chitin utilization pathway in the Vibrionaceae. Appl. Environ. Microbiol. 74, 44-51. doi: 10.1128/AEM.01412-07

Hyatt, D., Chen, G.-L., Locascio, P. F., Land, M. L., Larimer, F. W., and Hauser, L. J. (2010). Prodigal: prokaryotic gene recognition and translation initiation site identification. BMC Bioinformatics 11:119. doi: 10.1186/1471-2 105-11-119

Jiao, N., and Azam, F. (2011). "Microbial carbon pump and its significance for carbon sequestration in the ocean," in Microbial Carbon Pump in the Ocean, Vol. 10, eds N. Jiao, F. Azam, and S. Sanders (Washington, DC: Science/AAAS), 43-45.

Jurkevitch, E. (2007). Predatory behaviors in bacteria-diversity and transitions. Microbe Am. Soc. Microbiol. 2:67. doi: 10.1128/microbe.2.67.1

Kimes, N. E., López-Pérez, M., Flores-Félix, J. D., Ramírez-Bahena, M.-H., Igual, J. M., Peix, A., et al. (2015). Pseudorhizobium pelagicum gen. nov., sp. nov. isolated from a pelagic Mediterranean zone. Syst. Appl. Microbiol. 38, 293-299. doi: $10.1016 /$ j.syapm.2015.05.003

Kiørboe, T., and Jackson, G. A. (2001). Marine snow, organic solute plumes, and optimal chemosensory behavior of bacteria. Limnol. Oceanogr. 46, 1309-1318. doi: 10.4319/lo.2001.46.6.1309
Klippel, B., Lochner, A., Bruce, D. C., Davenport, K. W., Detter, C., Goodwin, L. A., et al. (2011). Complete genome sequences of krokinobactersp. Strain $4 \mathrm{H}-$ 3-7-5 and Lacinutrixsp. Strain 5H-3-7-4, Polysaccharide-degrading members of the family Flavobacteriaceae. J. Bacteriol. 193, 4545-4546. doi: 10.1128/JB.0 5518-11

Lassmann, T., and Sonnhammer, E. L. (2005). Kalign-an accurate and fast multiple sequence alignment algorithm. BMC Bioinformat. 6:298. doi: 10.1186/14712105-6-298

Lavigne, H., D’ortenzio, F., Ribera D’alcalà, M., Claustre, H., Sauzède, R., and Gacic, M. (2015). On the vertical distribution of the chlorophyll a concentration in the Mediterranean Sea: a basin scale and seasonal approach. Biogeosci. Discuss. 12, 4139-4181. doi: 10.5194/bgd-12-4139-2015

Longford, S. R., Tujula, N. A., Crocetti, G. R., Holmes, A. J., Holmström, C., Kjelleberg, S., et al. (2007). Comparisons of diversity of bacterial communities associated with three sessile marine eukaryotes. Aquat. Microbial. Ecol. 48, 217-229. doi: 10.3354/ame048217

Lowe, T. M., and Eddy, S. R. (1997). tRNAscan-SE: a program for improved detection of transfer RNA genes in genomic sequence. Nucleic Acids Res. 25, 955-964. doi: 10.1093/nar/25.5.0955

Mackelprang, R., Waldrop, M. P., Deangelis, K. M., David, M. M., Chavarria, K. L., Blazewicz, S. J., et al. (2011). Metagenomic analysis of a permafrost microbial community reveals a rapid response to thaw. Nature $480,368-371$. doi: 10.1038/nature10576

Mizuno, C. M., Rodriguez-Valera, F., and Ghai, R. (2015). Genomes of Planktonic Acidimicrobiales: Widening Horizons for Marine Actinobacteria by Metagenomics. MBio 6, e02083-e02014. doi: 10.1128/mBio.02083-14

Mizuno, C. M., Rodriguez-Valera, F., Kimes, N. E., and Ghai, R. (2013). Expanding the marine virosphere using metagenomics. PLoS Genet. 9:e1003987. doi: 10.1371/journal.pgen.1003987

Nawrocki, E. P. (2009). Structural RNA Homology Search and Alignment Using Covariance Models. Ph.D. thesis, Washington University School of Medicine, St. Louis, USA.

Parks, D. H., Imelfort, M., Skennerton, C. T., Hugenholtz, P., and Tyson, G. W. (2015). CheckM: assessing the quality of microbial genomes recovered from isolates, single cells, and metagenomes. Genome Res. 25, 1043-1055. doi: 10.1101/gr.186072.114

Peng, Y., Leung, H. C., Yiu, S.-M., and Chin, F. Y. (2012). IDBA-UD: a de novo assembler for single-cell and metagenomic sequencing data with highly uneven depth. Bioinformatics 28, 1420-1428. doi: 10.1093/bioinformatics/b ts174

Preheim, S. P., Boucher, Y., Wildschutte, H., David, L. A., Veneziano, D., Alm, E. J., et al. (2011). Metapopulation structure of Vibrionaceae among coastal marine invertebrates. Environ. Microbiol. 13, 265-275. doi: 10.1111/j.14622920.2010.02328.x

Price, M. N., Dehal, P. S., and Arkin, A. P. (2010). FastTree 2-approximately maximum-likelihood trees for large alignments. PLoS ONE 5:e9490. doi: 10.1371/journal.pone.0009490

Qin, J., Li, R., Raes, J., Arumugam, M., Burgdorf, K. S., Manichanh, C., et al. (2010). A human gut microbial gene catalogue established by metagenomic sequencing. Nature 464, 59-65. doi: 10.1038/nature08821

Quaiser, A., Zivanovic, Y., Moreira, D., and López-García, P. (2011). Comparative metagenomics of bathypelagic plankton and bottom sediment from the Sea of Marmara. ISME J. 5, 285-304. doi: 10.1038/ismej.2010.113

Raes, J., Korbel, J. O., Lercher, M. J., Von Mering, C., and Bork, P. (2007) Prediction of effective genome size in metagenomic samples. Genome Biol. 8:R10. doi: 10.1186/gb-2007-8-1-r10

Rendulic, S., Jagtap, P., Rosinus, A., Eppinger, M., Baar, C., Lanz, C., et al. (2004). A predator unmasked: life cycle of Bdellovibrio bacteriovorus from a genomic perspective. Science 303, 689-692. doi: 10.1126/science.1093027

Richards, G. P., Watson, M. A., Boyd, E. F., Burkhardt, W., Lau, R., Uknalis, J., et al. (2013). Seasonal levels of the Vibrio predator bacteriovorax in atlantic, pacific, and gulf coast seawater. Int. J. Microbiol. 2013:375371. doi: $10.1155 / 2013 / 375371$

Rieck, A., Herlemann, D. P., Jürgens, K., and Grossart, H.-P. (2015). Particleassociated differ from free-living Bacteria in surface waters of the Baltic Sea. Front. Microbiol. 6. doi: 10.3389/fmicb.2015.01297

Rocap, G., Larimer, F. W., Lamerdin, J., Malfatti, S., Chain, P., Ahlgren, N. A., et al. (2003). Genome divergence in two Prochlorococcus ecotypes 
reflects oceanic niche differentiation. Nature 424, 1042-1047. doi: 10.1038/ nature 01947

Rusch, D. B., Halpern, A. L., Sutton, G., Heidelberg, K. B., Williamson, S., Yooseph, S., et al. (2007). The Sorcerer II global ocean sampling expedition: northwest Atlantic through eastern tropical Pacific. PLoS Biol. 5:e77. doi: 10.1371/journal.pbio.0050077

Sano, E., Carlson, S., Wegley, L., and Rohwer, F. (2004). Movement of viruses between biomes. Appl. Environ. Microbiol. 70, 5842-5846. doi: 10.1128/AEM.70.10.5842-5846.2004

Santamaría, R. I., Bustos, P., Sepúlveda-Robles, O., Lozano, L., Rodríguez, C., Fernández, J. L., et al. (2014). Narrow-host-range bacteriophages that infect Rhizobium etli associate with distinct genomic types. Appl. Environ. Microbiol. 80, 446-454. doi: 10.1128/AEM.02256-13

Satinsky, B. M., Crump, B. C., Smith, C. B., Sharma, S., Zielinski, B. L., Doherty, M., et al. (2014). Microspatial gene expression patterns in the Amazon River Plume. Proc. Natl. Acad. Sci. 111, 11085-11090. doi: 10.1073/pnas.1402 782111

Segata, N., Waldron, L., Ballarini, A., Narasimhan, V., Jousson, O., and Huttenhower, C. (2012). Metagenomic microbial community profiling using unique clade-specific marker genes. Nat. Methods 9, 811-814. doi: 10.1038/nmeth.2066

Short, C. M., and Suttle, C. A. (2005). Nearly identical bacteriophage structural gene sequences are widely distributed in both marine and freshwater environments. Appl. Environ. Microbiol. 71, 480-486. doi: 10.1128/AEM.71.1.480-486.2005

Smedile, F., Messina, E., La Cono, V., Tsoy, O., Monticelli, L. S., Borghini, M., et al. (2013). Metagenomic analysis of hadopelagic microbial assemblages thriving at the deepest part of Mediterranean Sea, Matapan-Vavilov Deep. Environ. Microbiol. 15, 167-182. doi: 10.1111/j.1462-2920.2012.02827.x

Smith, M. W., Allen, L. Z., Allen, A. E., Herfort, L., and Simon, H. M. (2013). Contrasting genomic properties of free-living and particle-attached microbial assemblages within a coastal ecosystem. Front. Microbiol. 4:120. doi: 10.3389/fmicb.2013.00120

Sogin, M. L., Morrison, H. G., Huber, J. A., Welch, D. M., Huse, S. M., Neal, P. R., et al. (2006). Microbial diversity in the deep sea and the underexplored "rare biosphere". Proc. Natl. Acad. Sci. 103, 12115-12120. doi: 10.1073/pnas.0605127103
Sunagawa, S., Coelho, L. P., Chaffron, S., Kultima, J. R., Labadie, K., Salazar, G., et al. (2015). Structure and function of the global ocean microbiome. Science 348:1261359. doi: 10.1126/science.1261359

Tatusov, R. L., Natale, D. A., Garkavtsev, I. V., Tatusova, T. A., Shankavaram, U. T., Rao, B. S., et al. (2001). The COG database: new developments in phylogenetic classification of proteins from complete genomes. Nucleic Acids Res. 29, 22-28. doi: $10.1093 /$ nar/29.1.22

Temperton, B., and Giovannoni, S. J. (2012). Metagenomics: microbial diversity through a scratched lens. Curr. Opin. Microbiol. 15, 605-612. doi: 10.1016/j.mib.2012.07.001

Thompson, L. R., Field, C., Romanuk, T., Ngugi, D., Siam, R., Dorry, H., et al. (2013). Patterns of ecological specialization among microbial populations in the Red Sea and diverse oligotrophic marine environments. Ecol. Evol. 3, 1780-1797. doi: 10.1002/ece3.593

Wilkins, D., Yau, S., Williams, T. J., Allen, M. A., Brown, M. V., Demaere, M. Z., et al. (2013). Key microbial drivers in Antarctic aquatic environments. FEMS Microbiol. Rev. 37, 303-335. doi: 10.1111/1574-6976.12007

Williams, H. N., Lymperopoulou, D. S., Athar, R., Chauhan, A., Dickerson, T. L., Chen, H., et al. (2016). Halobacteriovorax, an underestimated predator on bacteria: potential impact relative to viruses on bacterial mortality. ISME J. 10, 491-499. doi: 10.1038/ismej.2015.129

Williams, H. N., Turng, B.-F., and Kelley, J. I. (2009). Survival response of Bacteriovorax in surface biofilm versus suspension when stressed by extremes in environmental conditions. Microb. Ecol. 58, 474-484. doi: 10.1007/s00248009-9499-7

Conflict of Interest Statement: The authors declare that the research was conducted in the absence of any commercial or financial relationships that could be construed as a potential conflict of interest.

Copyright (c) 2016 López-Pérez, Kimes, Haro-Moreno and Rodriguez-Valera. This is an open-access article distributed under the terms of the Creative Commons Attribution License (CC BY). The use, distribution or reproduction in other forums is permitted, provided the original author(s) or licensor are credited and that the original publication in this journal is cited, in accordance with accepted academic practice. No use, distribution or reproduction is permitted which does not comply with these terms. 